\title{
Trapped atomic condensates with anisotropic interactions
}

\author{
S. Yi and L. You \\ School of Physics, Georgia Institute of Technology, Atlanta, GA 30332-0430
}

(September 11, 2018)

\begin{abstract}
We study the ground state properties of trapped atomic condensates with electric field induced dipole-dipole interactions. A rigorous method for constructing the pseudo potential in the spirit of ladder approximation is developed for general non-spherical (polarized) particles interacting anisotropically. We discuss interesting features not previously considered for currently available alkali condensates. In addition to provide a quantitative assessment for controlling atomic interactions with electric fields, our investigation may also shed new light into the macroscopic coherence properties of the Bose-Einstein condensation (BEC) of dilute interacting atoms.
\end{abstract}

03.75.Fi,34.10.+x,32.80.Cy

The success of atomic Bose-Einstein condensation (BEC) [1] has stimulated great interest in the properties of trapped quantum gases. In standard treatments of interacting quantum gases, realistic inter-atomic potentials $V(\vec{R})$ are replaced by contact forms $u_{0} \delta(\vec{R})$ in the so-called shape independent approximation (SIA) [5]. Such an approximation results in tremendous simplification. To date, the SIA has worked remarkably well as recent theoretical investigations [4 have successfully accounted for almost all experimental observations [6, [7].

Currently available degenerate quantum gases are cold and dilute, with interactions dominated by low energy binary collisions. When realistic interatomic potentials are assumed to be isotropic and short ranged, i.e. decreasing faster than $-1 / R^{3}$ asymptotically for large interatomic separations $R$, the properties of a complete two body collision is described by just one atomic parameter: $a_{\mathrm{sc}}$, the s-wave scattering length. The scattering amplitude is isotropic and energy-independent: $f\left(\vec{k}, \vec{k}^{\prime}\right)=-4 \pi a_{\mathrm{sc}}$ for collisions involving incident momentum $\vec{k}$ scattering into $\vec{k}^{\prime}$. Effective physical mechanisms exist for control of the atom scattering lengths [8-10]. If implemented, these control 'knobs' allow for unprecedented comparison between theory and experiment over a wide range of interaction strength. Indeed, very recently several groups have successfully implemented Feshbach resonance [11], thus enabling a control knob on $a_{\mathrm{sc}}$ through the changing of an external magnetic field. Other physical mechanisms also exist for modifying atom-atom interactions, e.g. the shape resonance due to anisotropic dipole interactions inside an external electric field $[12$.

Although fermions with anisotropic interactions are well studied within the context of ${ }^{3} \mathrm{He}$ fluid [13] and in dwave high $T_{c}$ superconductors, anisotropically interacting bosons have not been studied in great detail. In particular, we are not aware of any systematic approach for constructing an anistropic pseudo potential [5].

In this paper, we study the ground state properties of trapped condensates with dipole interactions. A rigorous method is developed for constructing the anisotropic pseudo potential that can also be applied to future polar molecular BEC [14,15]. This Letter is organized as 
follows. First we briefly review the SIA pseudo-potential approximation. We then construct an analogous effective low energy anisotropic pseudo-potential. Numerical results are then discussed for ${ }^{87} \mathrm{Rb}[$ [1] inside the external E-field in the JILA TOP trap. We conclude with a brief discussion of prospects for realistic experiments.

For $N$ trapped spinless bosonic atoms in a potential $V_{t}(\vec{r})$, the second quantized Hamiltonian is given by

$$
\begin{aligned}
\mathcal{H}= & \int d \vec{r} \hat{\Psi}^{\dagger}(\vec{r})\left[-\frac{\hbar^{2}}{2 M} \nabla^{2}+V_{t}(\vec{r})-\mu\right] \hat{\Psi}(\vec{r}) \\
& +\frac{1}{2} \int d \vec{r} \int d \vec{r}^{\prime} \hat{\Psi}^{\dagger}(\vec{r}) \hat{\Psi}^{\dagger}\left(\vec{r}^{\prime}\right) V\left(\vec{r}-\vec{r}^{\prime}\right) \hat{\Psi}\left(\vec{r}^{\prime}\right) \hat{\Psi}(\vec{r}),
\end{aligned}
$$

where $\hat{\Psi}(\vec{r})$ and $\hat{\Psi}^{\dagger}(\vec{r})$ are atomic (bosonic) annihilation and creation fields. The chemical potential $\mu$ guarantees the atomic number $\hat{N}=\int d \vec{r} \hat{\Psi}^{\dagger}(\vec{r}) \hat{\Psi}(\vec{r})$ conservation.

The bare potential $V(\vec{R})$ in (1) needs to be renormalized for a meaningful perturbation calculation. For bosons, the usual treatment is based on field theory and is rather involved [5, 16 18]. Physically the SIA can be viewed as a valid low energy and low density renormalization scheme. The physics involved is rather simple: one simply replaces the bare potential $V(\vec{R})$ by the pseudo potential $u_{0} \delta(\vec{R})$ such that whose first order Born scattering amplitude reproduces the complete scattering amplitude $\left(-a_{\mathrm{sc}}\right)$. This requires $u_{0}=4 \pi \hbar^{2} a_{\mathrm{sc}} / M$.

When an electric field is introduced along the positive $\mathrm{z}$ axis, an additional dipole interaction

$$
V_{E}(\vec{R})=-u_{2} \frac{Y_{20}(\hat{R})}{R^{3}},
$$

appears, where $u_{2}=4 \sqrt{(\pi / 5)} \alpha(0) \alpha^{*}(0) \mathcal{E}^{2}$, with $\alpha(0)$ being the polarizability, and $\mathcal{E}$ the electric field strength. As was shown in Ref. [12], this modification results in a completely new low-energy scattering amplitude

$$
\left.f\left(\vec{k}, \vec{k}^{\prime}\right)\right|_{k=k^{\prime} \rightarrow 0}=4 \pi \sum_{l m, l^{\prime} m^{\prime}} t_{l m}^{l^{\prime} m^{\prime}}(\mathcal{E}) Y_{l m}^{*}(\hat{k}) Y_{l^{\prime} m^{\prime}}\left(\hat{k}^{\prime}\right),
$$

with $t_{l m}^{l^{\prime} m^{\prime}}(\mathcal{E})$ the reduced T-matrix elements. They are all energy independent and act as generalized scattering lengths. The anisotropic $V_{E}$ causes the dependence on both incident and scattered directions: $\hat{k}$ and $\hat{k}^{\prime}=\hat{R}$.

A general anisotropic pseudo potential can be constructed according to

$$
V_{\mathrm{eff}}(\vec{R})=u_{0} \delta(\vec{R})+\sum_{l_{1}>0, m_{1}} \gamma_{l_{1} m_{1}} \frac{Y_{l_{1} m_{1}}(\hat{R})}{R^{3}}
$$

whose first Born amplitude is then given by

$f_{\text {Born }}\left(\vec{k}, \vec{k}^{\prime}\right)=-(4 \pi)^{2} a_{\mathrm{sc}} Y_{00}^{*}(\hat{k}) Y_{00}\left(\hat{k}^{\prime}\right)-\frac{M}{4 \pi \hbar^{2}} \sum_{l_{1} m_{1}} \gamma_{l_{1} m_{1}}(4 \pi)^{2} \sum_{l m} \sum_{l^{\prime} m^{\prime}} \mathcal{T}_{l m}^{l^{\prime} m^{\prime}}\left(l_{1}, m_{1}\right) Y_{l m}^{*}(\hat{k}) Y_{l^{\prime} m^{\prime}}\left(\hat{k}^{\prime}\right)$,

with $\mathcal{T}_{l m}^{l^{\prime} m^{\prime}}\left(l_{1}, m_{1}\right)=(i)^{l+l^{\prime}} \mathcal{R}_{l}^{l^{\prime}} I_{l m}^{l^{\prime} m^{\prime}}\left(l_{1}, m_{1}\right)$. Both 


$$
\begin{aligned}
I_{l m}^{l^{\prime} m^{\prime}}\left(l_{1} m_{1}\right) & =\left\langle Y_{l^{\prime} m^{\prime}}\left|Y_{l_{1} m_{1}}\right| Y_{l m}\right\rangle, \quad \text { and } \\
\mathcal{R}_{l}^{l^{\prime}} & =\int_{0}^{\infty} d R \frac{1}{R} j_{l}(k R) j_{l^{\prime}}\left(k^{\prime} R\right),
\end{aligned}
$$

can be computed analytically [20]. The $1 / R^{3}$ form in Eq. (四) assures all $\mathcal{R}_{l}^{l^{\prime}}$ to be $k=k^{\prime}$ independent [by a change of variable to $x=k R$ in the integral]. Putting

$$
f_{\text {Born }}\left(\vec{k}, \vec{k}^{\prime}\right)=f\left(\vec{k}, \vec{k}^{\prime}\right),
$$

one can solve for the $\gamma_{l_{1} m_{1}}(\mathcal{E})$ as $t_{l m}^{l^{\prime} m^{\prime}}(\mathcal{E})$ are known numerically [12, 19]. This reduces to the linear equations

$$
-\frac{M}{4 \pi \hbar^{2}} \sum_{l_{1} m_{1}} \gamma_{l_{1} m_{1}}(4 \pi) \mathcal{T}_{l m}^{l^{\prime} m^{\prime}}\left(l_{1}, m_{1}\right) \equiv t_{l m}^{l^{\prime} m^{\prime}}
$$

for all $(l m)$ and $\left(l^{\prime} m^{\prime}\right)$ with $l, l^{\prime} \neq 0$, and separately $a_{\mathrm{sc}}(\mathcal{E})=-t_{00}^{00}(\mathcal{E})$. The problem simplifies further for Bosons (fermions) as only even (odd) $\left(l, l^{\prime}\right)$ terms are needed to match. Figure 1 displays result of $a_{\mathrm{sc}}(\mathcal{E})$ for the triplet state of ${ }^{87} \mathrm{Rb}$. The Born amplitude for the dipole term $V_{E}$ is

$f_{\text {Born }}\left(\vec{k}, \vec{k}^{\prime}\right)=u_{2} \frac{M}{4 \pi \hbar^{2}}(4 \pi)^{2} \mathcal{T}_{00}^{20} \sum_{l m, l^{\prime} m^{\prime}} \overline{\mathcal{T}}_{l m}^{l^{\prime} m^{\prime}} Y_{l m}^{*}(\hat{k}) Y_{l^{\prime} m^{\prime}}\left(\hat{k}^{\prime}\right)$,

with $\mathcal{T}_{00}^{20}=-0.023508 . \overline{\mathcal{T}}_{l m}^{l^{\prime} m^{\prime}}=\mathcal{T}_{l m}^{l^{\prime} m^{\prime}}(2,0) / \mathcal{T}_{00}^{20}$ are tabulated below for small $\left(l, l^{\prime}\right)$.

TABLE I. $\overline{\mathcal{T}}_{l m}^{l^{\prime} m^{\prime}}$

\begin{tabular}{c|ccccc}
\hline \hline$(l m),\left(l^{\prime} m^{\prime}\right)$ & $(00)$ & $(20)$ & $(40)$ & $(60)$ & $(80)$ \\
\hline$(00)$ & 0 & 1 & 0 & 0 & 0 \\
$(20)$ & 1 & -0.63889 & 0.14287 & 0 & 0 \\
$(40)$ & 0 & 0.14287 & -0.17420 & 0.05637 & 0 \\
$(60)$ & 0 & 0 & 0.05637 & -0.08131 & 0.03008 \\
$(80)$ & 0 & 0 & 0 & 0.03008 & -0.04707 \\
\hline \hline
\end{tabular}


We found that away from shape resonances, Table (II) agrees $(\sim$ a few per cent) with the same ratios $t_{l m}^{l^{\prime} m^{\prime}}(\mathcal{E}) / t_{00}^{20}(\mathcal{E})$ from the numerical multi-channel calculations 12. This interesting observation applies for all bosonic alkali triplet states we computed: ${ }^{7} \mathrm{Li},{ }^{39,41} \mathrm{~K}$, and ${ }^{85,87} \mathrm{Rb}$, for up to a field strength of $3 \times 10^{6}(\mathrm{~V} / \mathrm{cm})$ [12,19. Physically, this implies that effect of $V_{E}$ is perturbative as $\mathcal{E}$ remains small in atomic units. What is remarkable is that $\mathcal{T}_{00}^{20}(\mathcal{E})$ and $t_{00}^{20}(\mathcal{E})$ also agree in absolute values 12 . For ${ }^{87} \mathrm{Rb}$, we found

$$
u_{2} \frac{M}{4 \pi \hbar^{2}}(4 \pi)^{2} \mathcal{T}_{00}^{20}=-1.495 \times 10^{10} \overline{\mathcal{E}}^{2}\left(a_{0}\right),
$$

with $\overline{\mathcal{E}}$ in atomic units $\left(5.142 \times 10^{9} \mathrm{~V} / \mathrm{cm}\right) . \quad a_{0}$ is the Bohr radius. While multi-channel scattering gives 19

$$
(4 \pi) t_{00}^{20}=-1.512 \times 10^{10} \overline{\mathcal{E}}^{2}\left(a_{0}\right) .
$$

The cause of this slight difference $(1 \%)$ is not entirely clear and but is within numerical error.

We can thus approximate Eq. (4) by keeping only the $l_{1}=2, m_{1}=0$ term in the sum

$$
V_{\text {eff }}(\vec{R})=u_{0} \delta(\vec{R})-u_{2} Y_{20}(\hat{R}) / R^{3},
$$

away from the shape resonance. At zero temperature the condensate wave function $\psi(\vec{r}, t)=\langle\hat{\Psi}(\vec{r}, t)\rangle$ then obeys the following nonlinear Schrodinger equation

$$
\begin{aligned}
i \hbar \frac{d}{d t} \psi(\vec{r}, t) & =\left[-\frac{\hbar^{2}}{2 M} \nabla^{2}+V_{t}(\vec{r})-\mu+u_{0}|\psi(\vec{r}, t)|^{2}\right. \\
& \left.-u_{2} \int d \vec{r}^{\prime} \frac{Y_{20}(\hat{R})}{R^{3}}\left|\psi\left(\vec{r}^{\prime}, t\right)\right|^{2}\right] \psi(\vec{r}, t),
\end{aligned}
$$

with $\psi(\vec{r}, t)$ normalized to $N$. The ground state is found by steepest descent through propagation of Eq. (12) in imaginary time $(i t)$. For a cylindrical symmetric trap $V_{\mathrm{t}}(\vec{r})=M\left(\omega_{\perp}^{2} x^{2}+\omega_{\perp}^{2} y^{2}+\omega_{z}^{2} z^{2}\right) / 2$, the ground state also possesses azimuthal symmetry. Therefore the non-local term simplifies to

$$
\int d \vec{r}^{\prime}\left|\psi\left(\rho^{\prime}, z^{\prime}\right)\right|^{2} \frac{Y_{20}(\hat{R})}{R^{3}}=\int d z^{\prime} d \rho^{\prime} \mathcal{K}(., . ; .)\left|\psi\left(\rho^{\prime}, z^{\prime}\right)\right|^{2},
$$

with the kernel $\mathcal{K}\left(\rho, \rho^{\prime} ; z-z^{\prime}\right)$ expressed in terms of the standard Elliptical integrals E[.] and K[.]. The kernel is divergent at $\vec{r}=\vec{r}^{\prime}$, so a cut-off radius $R_{c}$ is chosen such that $\mathcal{K}\left(\rho^{\prime}, \rho, z^{\prime}-z\right)=0$ whenever $\left|\vec{r}-\vec{r}^{\prime}\right|<R_{c}$. We typically $R_{c} \sim 50\left(a_{0}\right)$, much smaller than the grid size, to minimize numerical errors. Technical details for numerical computations and for handling the singular rapid variation of the kernel over small length scale will be discussed elsewhere 20].

Figure 2 presents $\psi(\rho, z)$ along $\rho=0$ (a) and $z=0$ (b) cuts respectively for ${ }^{87} \mathrm{Rb}\left(a_{\mathrm{sc}}=5.4 \mathrm{~nm}\right)$ at several different $\mathcal{E}$. We note the condensate shrinks radially 
while stretches along z-axis to minimize the dipole interaction $V_{E}$. The top-right corner inserts shows electric field polarized atoms in (radially) repulsive (a) and (longitudinally) attractive (b) configurations. An elongated condensate along the $\mathrm{z}$-axis reduces the total energy. The same mechanism could cause spontaneous alignment of polar molecular condensates inside isotropic traps [14]. For better insights we try a variation ansatz

$$
\psi_{T}(\rho, z)=\frac{\kappa^{1 / 2}}{\pi^{3 / 4} d^{3 / 2}} \exp \left[-\frac{1}{2 d^{2}}\left(\rho^{2}+\kappa^{2} z^{2}\right)\right],
$$

with parameters $d$ and $\kappa$. In dimensionless units for length $\left(a_{\perp}=\sqrt{\hbar / M \omega_{\perp}}\right)$, energy $\left(\hbar \omega_{\perp}\right)$, and $\lambda=\omega_{z} / \omega_{\perp}$, we obtain

$E\left[\psi_{T}\right]=\left(1+\frac{\lambda^{2}}{2 \kappa^{2}}\right) d^{2}+\left(1+\frac{\kappa^{2}}{2}\right) \frac{1}{d^{2}}+\frac{4 N \kappa}{\sqrt{2 \pi}} \frac{a_{\mathrm{sc}}^{\mathrm{eff}}}{a_{\perp}} \frac{1}{d^{3}}$,

with the effective scattering length $a_{\mathrm{sc}}^{\mathrm{eff}}=a_{\mathrm{sc}}[1-$ $\left.b(\kappa) u_{2} / u_{0}\right]$, and

$$
b(\kappa)=\frac{\sqrt{5 \pi}}{3\left(\kappa^{2}-1\right)}\left(-2 \kappa^{2}-1+\frac{3 \kappa^{2} \tanh ^{-1} \sqrt{1-\kappa^{2}}}{\sqrt{1-\kappa^{2}}}\right) .
$$

The $b(\kappa)$ is monotonically decreasing, and bounded between $b(0)=\sqrt{5 \pi} / 3$ and $b(\infty)=-2 \sqrt{5 \pi} / 3 . a_{\mathrm{sc}}^{\text {eff }}$ is shown in Fig. 3 as a function of $\mathcal{E}$ for several different values of $\kappa$. For increasing electric field $\mathcal{E}$, variational calculation results in decreasing $\kappa$, eventually $\kappa$ becomes less than one, i.e. the condensate changes from oblate (pancake) shaped at zero field (for the TOP trap) to prolate (cigar) shaped. We also note that $b(\kappa) \geq 0$ for $\kappa \leq 1$, therefore $a_{\mathrm{sc}}^{\text {eff }}$ becomes negative at certain field value $\mathcal{E}_{c}$ in the case of a positive $a_{\mathrm{sc}}(\mathcal{E}=0)$, causing the collapse of the condensate. This is indeed what we found as illustrated in Fig. 4. A detailed discussion of the collapse and other interesting features will be given elsewhere [20].

We note the energy of dipole alignment

$$
E_{P} \sim-(2 \pi) 1 \times 10^{18} \times \overline{\mathcal{E}}^{2}(\mathrm{~Hz}),
$$

becomes much larger than the trap depth at the proposed $\mathcal{E}$ values for ${ }^{87} \mathrm{Rb}$. Therefore spatial homogeneity for $\mathcal{E}(\vec{r})$ is required. At $\mathcal{E} \sim 5 \times 10^{5}(\mathrm{~V} / \mathrm{cm})\left[\overline{\mathcal{E}} \sim 10^{-4}\right]$ with a spatial gradient $<10^{-4} / \mathrm{cm}^{3}$, the corresponding force is smaller than the magnetic trapping force for typical traps at $\sim 100(\mathrm{~Hz})$. For comparison, the magnetic field gradient is $\sim 10^{-6} / \mathrm{cm}^{3}$ inside the Penning trap magnets. Although the proposed electric field $\left[10^{5}(\mathrm{~V} / \mathrm{cm})<\mathcal{E}<10^{6}\right.$ $(\mathrm{V} / \mathrm{cm})]$ is large, it can be created through careful laboratory techniques as breakup is fundamentally limited by field ionization, which typically occurs at $\mathcal{E}>10^{7}(\mathrm{~V} / \mathrm{cm})$ [23]. Recently a $\mathcal{E}$ field of upto $1.25 \times 10^{5}(\mathrm{~V} / \mathrm{cm})$ was used successfully to decelerate a molecular beam [24].

In conclusion, we have developed a general scheme for constructing effective pseudo-potentials for anisotropic interactions. Our scheme guarantees that the first 
order Born scattering amplitude from the pseudopotential reproduces the complete scattering amplitude obtained from a multi-channel computation including the anisotropic dipole-interaction, thus contains no energy dependence at low temperatures of the trapped atomic gases [12]. Our scheme is thus more pleasing than the standard Skyrme type velocity dependent effective potentials commonly adopted in nuclear physics [25]. We also presented results for both the electric field modified atomic scattering parameters and the induced changes to the condensate for ${ }^{87} \mathrm{Rb}$ in the JILA TOP trap. Our theory can be directly extended to systems involving magnetic dipole interaction of atoms/molecules in a static magnetic trap and systems of trapped molecules with permanent electric dipoles [14]. For alkali atoms, typical magnetic dipole interaction is weak since a Bohr magneton $\left(\mu_{B}=e \hbar / 2 m c\right)$ only corresponds to an electric dipole of $\sim\left(1 / 2 \alpha_{f}\right)\left(e a_{0}\right)$ (fine structure constant $\left.\alpha_{f} \approx 1 / 137\right)$, which is equivalent to the induced electric dipole at $\mathcal{E}=6 \times 10^{4}(\mathrm{~V} / \mathrm{cm})$ for ${ }^{87} \mathrm{Rb}$. Other atoms with larger magnetic dipole moments 26 will display clearer anisotropic effects. Typical hetero-nuclear diatomic molecules have a permanent electric dipole moment of $\sim\left(e a_{0}\right)$, corresponding to an induced moment in ${ }^{87} \mathrm{Rb}$ at $\mathcal{E}=1.6 \times 10^{7}(\mathrm{~V} / \mathrm{cm})$ [14. Trapped molecules with aligned permanent electric dipoles (by an external E-field) would give similar results. However, magnetic trapped molecules [14] with unaligned electric dipoles interacting with the spin axis represents an interesting extension that requires further investigation.

We thank Dr. M. Marinescu for helpful discussions during the early stages of this work. This work is supported by the U.S. Office of Naval Research grant No. 14-97-1-0633 and by the NSF grant No. PHY-9722410.

[1] M. H. Anderson et al., Science 269, 198 (1995).

[2] K. B. Davis et al., Phys. Rev. Lett. 75, 3969 (1995).

[3] C. C. Bradley et al., Phys. Rev. Lett. 75, 1687 (1995); ibid 79, 1170 (1997).

[4] Extentive list of references exists at: http://amo.phy.gasou.edu/bec.html/bibliography.htm.

[5] Kerson Huang and C. N. Yang, Phys. Rev. 105, 767 (1957).

[6] M. Naraschewski and R.J. Glauber, Phys. Rev. A 59, 4595 (1999).

[7] B. Esry and C. Greene, Phys. Rev. A 60, 1451 (1999).

[8] P. O. Fedichev et al., Phys. Rev. Lett. 77, 2913 (1996); J. L. Bohn and P. S. Julienne, Phys. Rev. A 56, 1486 (1997).

[9] A. J. Moerdijk et al., Phys. Rev. A 53, 4343 (1996).

[10] E. Tiesinga et al., Phys. Rev. A 46, R1167 (1992).

[11] S. Inouye et. al, Nature (London) 392, 151 (1998); Ph. Courteille et. al, Phys. Rev. Lett. 81, 69 (1998); J. L. 
Roberts et. al, ibid 81, 5179 (1998); Vladan Vuletic et. al, ibid 82, 1406 (1999).

[12] M. Marinescu and L. You, Phys. Rev. Lett. 81, 4596 (1998).

[13] A. J. Leggett, Rev. Mod. Phys. 47, 331 (1975).

[14] J. D. Weinstein et. al, Nature 395, 148 (1998).

[15] J. T. Bahns et al.,(preprint, 1999).

[16] V. Galitskii, Sov. Phys. JETP 34, 104 (1958) [Zh. Eksp. Teor. Fiz. 34, 151 (1958)].

[17] S. T. Beliaev, Sov. Phys. JETP 7, 289 (1958) [Zh. Eksp. Teor. Fiz. 34, 417 (1958)]; N. M. Hugenholtz and D. Pines, Phys. Rev. 116, 489 (1959).

[18] K. A. Brueckner and K. Sawada, Phys. Rev. 106, 1117 (1957).

[19] M. Marinescu and L. You, (unpublished).

[20] S. Yi and L. You, (to be published).

[21] E. P. Gross, Nuovo Cimento 20, 454 (1961); L. Pitaevskii, Sov. Phys. JETP 13, 451 (1961).

[22] G. Baym and C. J. Pethick, Phys. Rev. Lett. 76, 6 (1996); Victor M. Perez-Garcia et al., ibid 77, 5320 (1996).

[23] R. V. Latham, High Voltage Vacuum Insulation: The physical basis, (Academic Press, New York, 1981); T. B. Mitchell, AIP Proceedings 331, 115 (1995); J. C. Davis, (private communications).

[24] H. L. Bethlem et al., Phys. Rev. Lett. 83, 1558 (1999).

[25] P. Ring and P. Schuck, The Nuclear Many-Body Problem, p. 172, (Springer-Verlag, New York, 1980).

[26] J. Weinstein et al., Phys. Rev. A 57, R3173 (1998).

FIG. 1. The field dependent value for $a_{\text {sc }}$. Note the shape resonance for $\mathcal{E}$ around $8.3 \times 10^{5}(\mathrm{~V} / \mathrm{cm})$.

FIG. 2. (a) $\psi(\rho, 0)$ for ${ }^{87} \mathrm{Rb}$ with $\omega_{\perp}=(2 \pi) 70(\mathrm{~Hz})$, $\omega_{z}=\sqrt{8} \omega_{\perp}$, and $N=5000$ atoms. Solid, dashed-dot, dashed, and dotted lines are for $\mathcal{E}=0,4.0 \times 10^{5}, 5.7 \times 10^{5}$, and $5.88 \times 10^{5}(\mathrm{~V} / \mathrm{cm})$ respectively. $a_{\perp}$ is the radial trap width. (b) same as in Fig. 2 (a), but for $\psi(0, z)$.

FIG. 3. Typical behavior of the effective scattering length $a_{\mathrm{sc}}^{\mathrm{eff}}$. Lines corresponds to $\kappa=5.1,1.7,1.02,0.34,0.017$, in descending order of $a_{\mathrm{sc}}^{\text {eff }}$.

FIG. 4. Electric field dependence of the width aspect ratio for parameters of Fig. 2. The solid line is the result of our variational calculation while circles denote exact numerical results. The dashed line corresponds to $\sqrt{\sqrt{8}}$, the result for a non-interacting gas in the TOP trap. 


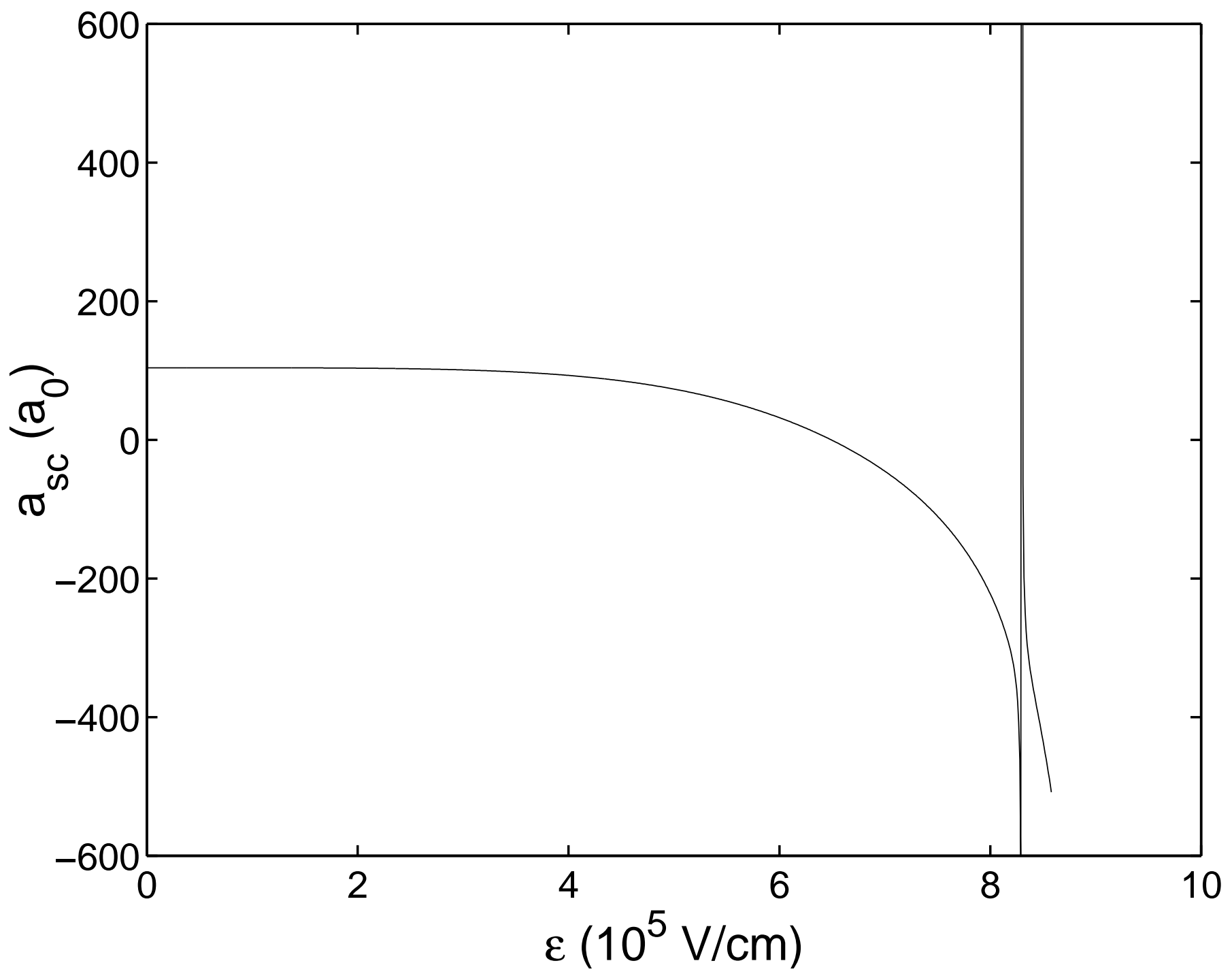




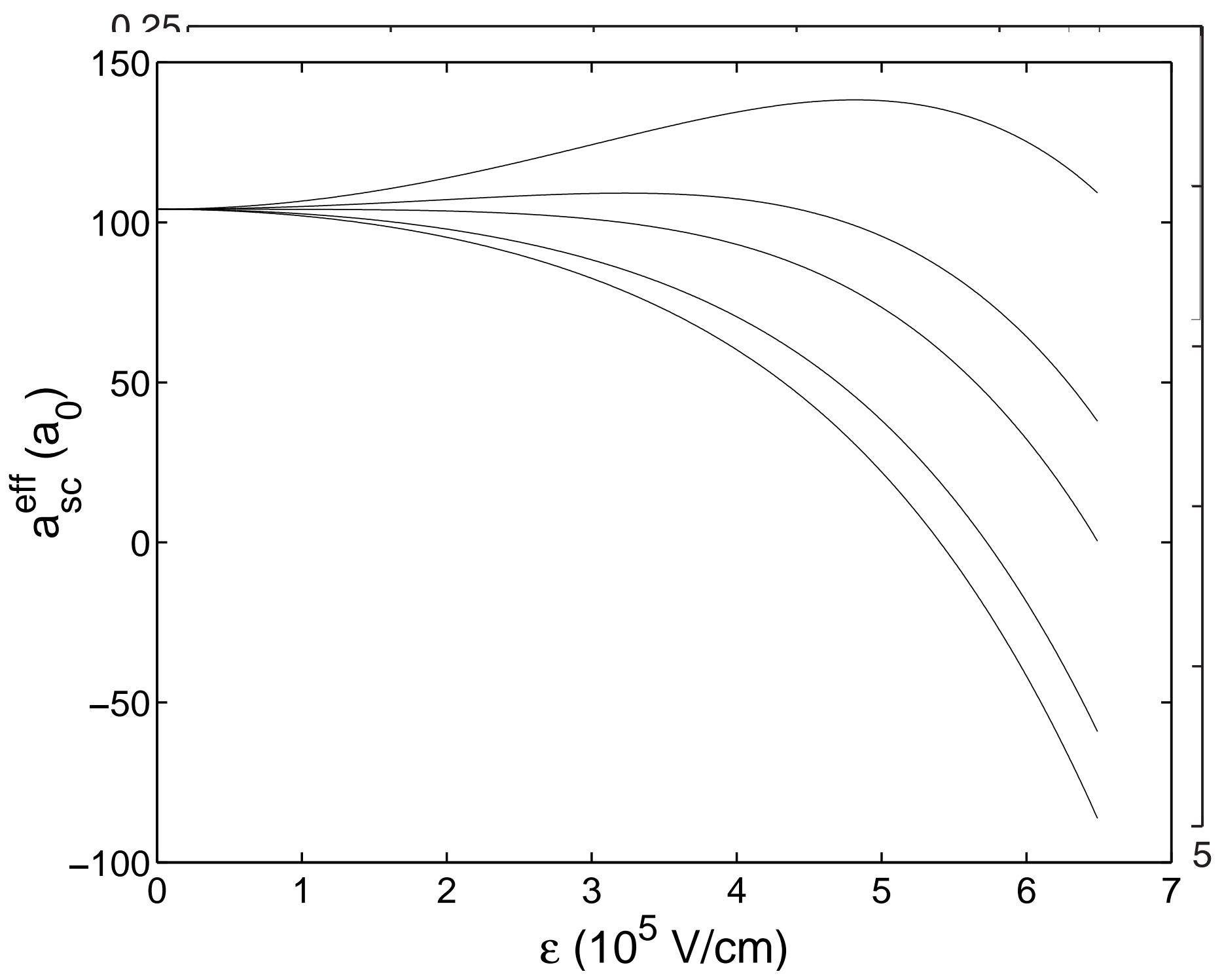




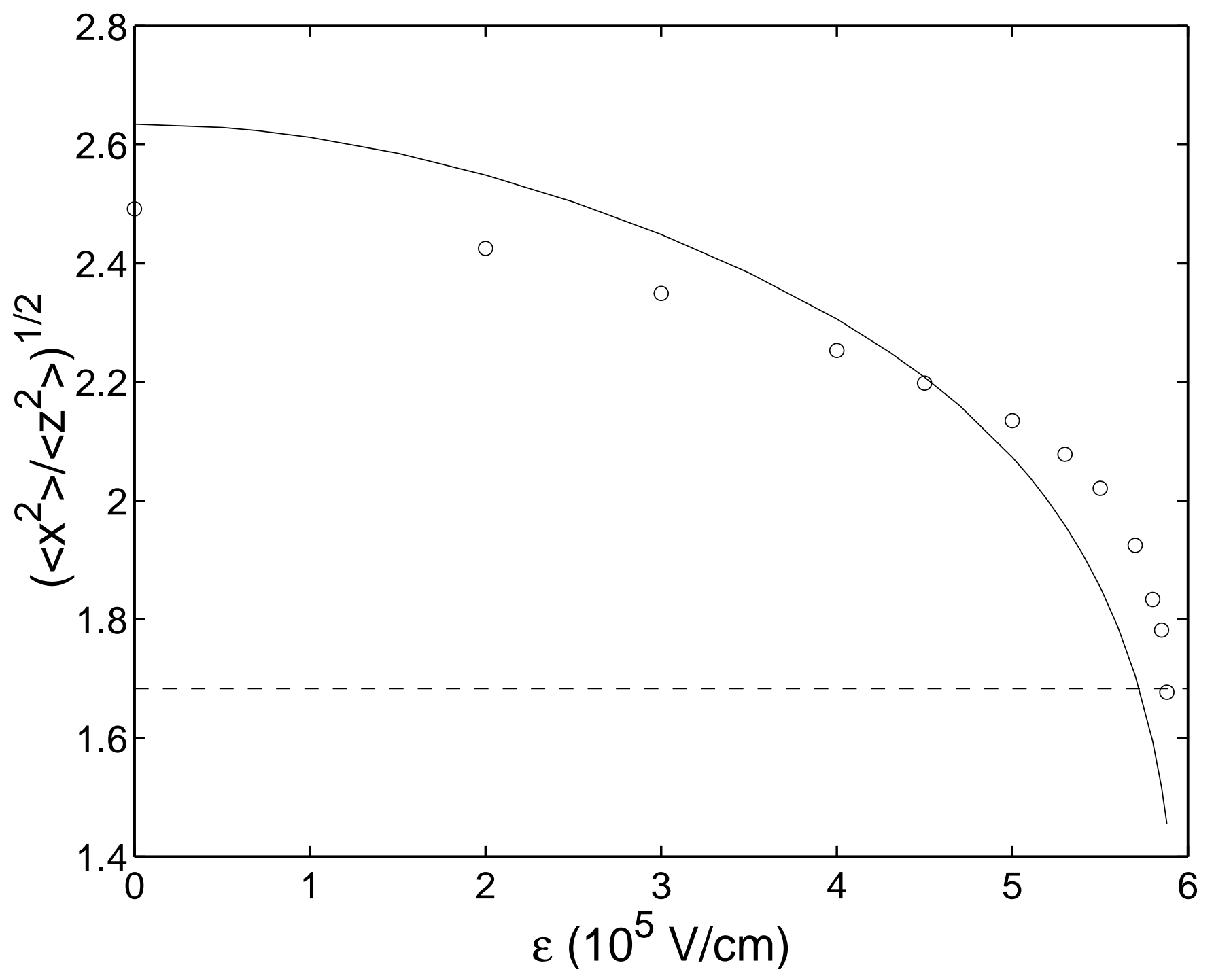

